Vitrinite reflectance data of cuttings $(3,540-11,850)$ from the Gulf 0 il Corp. Point McIntyre No. I well 


\begin{tabular}{|c|c|c|c|}
\hline Filename & Title & & \\
\hline FTMCOZ & GULF FT. & MCINTYKE & $3540-\$ 810^{\circ}$ \\
\hline Date & Time & & \\
\hline $01-15-1900$ & $02: 13: 35$ & & \\
\hline
\end{tabular}

Number of readings: 40

\begin{tabular}{|c|c|c|c|c|c|c|}
\hline \multicolumn{2}{|c|}{ Fieading \# } & +0 & +1 & +2 & $+\xi$ & +4 \\
\hline 1 & $=$ &. .31 & .26 & .28 & .23 & .38 \\
\hline 6 & $=$ & .23 & .57 & .55 & .52 & .38 \\
\hline 11 & $=$ & .5 & .3 & .47 & .5 & .34 \\
\hline 16 & $=$ & .35 & .39 & .52 & .38 & .49 \\
\hline 21 & $=$ & .47 & .29 & .31 & .28 & .39 \\
\hline 26 & $=$ & .52 & .24 & .27 & .26 & .43 \\
\hline 31 & $=$ & .32 & .49 & .45 &.$\$ 6$ & .26 \\
\hline 36 & $=$ & .52 & .34 & $.4 \bar{\theta}$ & .41 & .38 \\
\hline
\end{tabular}

Number of groups: 1

Group \# 1 left limit is : 0 Group \#1 right limit is : 10 


\begin{tabular}{|c|c|c|c|c|c|c|}
\hline \multirow{2}{*}{$\begin{array}{l}\text { Fil ename } \\
\text { FTMCO4 }\end{array}$} & \multicolumn{2}{|c|}{ Title } & \multirow[b]{2}{*}{ MCINTYFEE \#1 } & \multirow[b]{2}{*}{$4620-4890^{\circ}$} & & \\
\hline & GULF & FT. & & & & \\
\hline Date & Tin & & & & & \\
\hline $01-15-1980$ & $02: 48$ & $8: 59$ & & & & \\
\hline Number of & readings: & 35 & & & & \\
\hline Fieading \# & & +0 & +1 & +2 & +3 & +4 \\
\hline 1 & $=$ & 45 & .54 & 47 & .36 & .27 \\
\hline 6 & $=$ & .42 & .36 & .4 & .34 & .41 \\
\hline 11 & $=$ & 27 & .35 & .35 & .3 & .37 \\
\hline 16 & $=$ & .29 & .45 & .34 & .46 & .3 \\
\hline 21 & $=$ & 5 & .38 & .35 & .41 & .31 \\
\hline 26 & $=$ & 19 & .55 & .32 & .52 & .32 \\
\hline 31 & $=$ & . $\Xi$ &.$\$ 6$ & .35 & .39 & .32 \\
\hline
\end{tabular}

Number of groups: 1

Group \# 1 left limit is: 0

Group \#1 right 1 imit is : 10 
Filename

-ー-

F.TMCÖG

Date

----

$01-15-1900$

Number of readings: 40

\begin{tabular}{ll} 
Fieading \# & \\
\hdashline 1 & $=$ \\
6 & $=$ \\
11 & $=$ \\
16 & $=$ \\
21 & $=$ \\
26 & $=$ \\
31 &
\end{tabular}

Number of groups: 1
Group \#1 left limit is : 0

$\begin{array}{ll}+1 & +2 \\ -.28 & -28 \\ .25 & .28 \\ .25 & .29 \\ .31 & .42 \\ .58 & .35 \\ .32 & .26 \\ . .56 & .4 \\ .36 & .45\end{array}$

$+3$

$---$

.41

.34

.27

.47

. 30

.42

.38

.56
$+4$

27

.27

.27

.42

.34

.34

.35

.26

.38
Group \# 1 right limit is : 10 


\begin{tabular}{|c|c|c|c|c|c|c|}
\hline Filename & Tit & tle & & & & \\
\hline FTMCOB & GULF & FT. & MCINTYRE \#1 & $6780-7$ & & \\
\hline Date & Tin & me & & & & \\
\hline $01-15-1980$ & $05: 2$ & $0: 00$ & & & & \\
\hline Number of & readirigs & 30 & & & & \\
\hline Reading \# & & +0 & +1 & +2 & +3 & +4 \\
\hline 1 & $=$ & .43 & .42 & .34 & .33 & .38 \\
\hline 6 & $=$ & .39 & .37 & .38 & .51 & .48 \\
\hline 11 & $=$ & .36 & .58 & .48 & .35 & .3 \\
\hline 16 & $=$ & .38 & .36 & .33 & .36 & .39 \\
\hline 21 & $=$ & .43 & .4 & .41 & .44 & .4 \\
\hline 26 & $=$ & .36 & .56 & .32 & .42 & .35 \\
\hline 31 & $=$ & .2 & .56 & .4 & .4 & .26 \\
\hline
\end{tabular}

Number of groups: 1

Group \#1 left limit is: 0

Group \# 1 right limit is: 10

GMC Data Report No. 151

Page 4/8 
Filename

PTMC10

\begin{tabular}{lc} 
Date & Time \\
\hdashline $01-15-1980$ & O5:5ó:02
\end{tabular}

Number of readings: 40

\begin{tabular}{|c|c|c|c|c|c|c|}
\hline Fieading \# & & +0 & +1 & +2 & +3 & +4 \\
\hline 1 & $=$ & . & .24 & .41 & .31 & .33 \\
\hline 6 & $=$ & .34 & .38 & .37 & .36 & .29 \\
\hline 11 & $=$ & .23 & .34 & .59 & .46 & .33 \\
\hline 16 & $=$ & .22 & .57 & .32 & .27 & .49 \\
\hline 21 & $=$ &.$\Xi$ & . $\Xi 5$ & .35 & .45 & .39 \\
\hline 26 & $=$ & .4 & .47 & .27 & .42 & .28 \\
\hline 31 & $=$ & .35 & .29 & .43 & .38 & .39 \\
\hline 36 & $=$ & .25 &.$\square 4$ & .5 & .54 & .23 \\
\hline
\end{tabular}

Number of groups: 1

Group \#1 left limit is : 0

Group \#1 right 1 imit is : 10 
Fi 1 ename

FTMC12

Date

$01-15-1930$
Title

GULF FT. MCINTYFIE \#1 8940-9210\%

Time

06:14:06

Number of readings: $\$ 7$

\begin{tabular}{|c|c|c|c|c|c|c|}
\hline Reading & $\#$ & +0 & +1 & +2 & +3 & +4 \\
\hline 1 & $=$ & .43 & .36 & .48 & .43 & .4 \\
\hline 6 & $=$ & .4 & .41 &.$\$ 6$ & .42 & .46 \\
\hline 11 & $=$ & .41 & .39 & .42 & .27 & $.4 \Xi$ \\
\hline 16 & $=$ & .38 & .41 & .41 & .45 & .37 \\
\hline 21. & $=$ & .33 & .52 & .31 & .32 & .4 \\
\hline $26^{\circ}$ & $=$ & .5 & .4 & .37 & .44 & .38 \\
\hline 31 & $=$ & .36 & .5 & .48 & .42 & .42 \\
\hline
\end{tabular}

Number of groups: 1

Group \# 1 left limit is : 0

Group \# 1 right limit is : 10 
Filename

FTMC 13

Date

Date-

$01-15-1980$

Number of readings: $\Xi B$

\section{Reading \#}

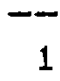

1

6

11

16

21

26

$\$ 1$

$\$ 6$
Title

GULF FT. MCINTYFE \#1 9480-9750"

Time

$-\cdots$

$06: 37: 28$

\begin{tabular}{|c|c|c|c|c|}
\hline+0 & +1 & +2 & +5 & +4 \\
\hline .36 & .51 & .42 & .44 & .4 \\
\hline .32 & .34 & .41 & .39 & .45 \\
\hline . $\$ 8$ & .23 & .58 & .44 & .37 \\
\hline .4 & .41 & .47 & . उ8 & .39 \\
\hline .4 & .29 & .35 & .35 & .44 \\
\hline .41 & .39 & .37 & .44 & .45 \\
\hline .37 & .39 & .42 & .55 & .47 \\
\hline .27 & .55 & .36 & & \\
\hline
\end{tabular}

Number of groups: 1

Group \# 1 left limit is: 0

Group \#1 right 1 imit is : 10

GMC Gata Report No. 151

Page 7/8 


\begin{tabular}{lc} 
Filename & Title \\
\hline PTMC17 & GULF FT MCINTYRE \#1 11580-11850" \\
Date & Time \\
\hline $01-15-1980$ & $-06: 55: 52$
\end{tabular}

\begin{tabular}{|c|c|c|c|c|c|c|}
\hline Reading & & $+\overline{0}$ & +1 & +2 & +5 & +4 \\
\hline 1 & $=$ & .51 & .4 & ---2 & 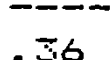 & .49 \\
\hline 6 & $=$ & .36 & .29 & .5 & .28 & .37 \\
\hline 11 & $=$ & .39 & . उడ & .47 & .4 & .41 \\
\hline 16 & $=$ & .46 & .35 & .41 & .39 & .25 \\
\hline 21 & $=$ & .28 & .32 & .46 & .29 & .38 \\
\hline 26 & $=$ & .4 .5 & .32 & .35 & .52 & .36 \\
\hline 31 & $=$ & .37 & .41 & .42 & .5 & .41 \\
\hline
\end{tabular}

Number of groups: 1

Group \# 1 left limit is: 0

Group \#1 right limit is: 10 